\section{Astronomical significance of sun-dials in Srirangapatna, Karnataka, India}

\author{
Aalok Pandya ${ }^{1,4, *}$, Tej Bahadur ${ }^{2}$ and \\ Sandip Bhattacharya $a^{3,4}$ \\ ${ }^{1}$ Department of Physics, JECRC University, Jaipur 303 905, India \\ ${ }^{2}$ Manipal University Jaipur 303 007, India \\ ${ }^{3}$ B.M. Birla Planetarium, Statue Circle, Jaipur 302 001, India \\ ${ }^{4}$ Astronomy and Astrophysics Resource Development and Research \\ Academy (AARDRA), Jaipur 302 017, India
}

This article reports astronomical significance of two special sun-dials installed by Tipu Sultan in Srirangapatna in Karnataka, India. One sun-dial is situated in Jamia Masjid, a prominent mosque and the other is situated in Hyder Ali's tomb, popularly known as Gumbaz. These sun-dials are very different from most known sun-dials in design and concept. Both the sundials are quite identical. The astronomical idea upon which these sun-dials were designed are explored and reported here.

Keywords: Analema, local time, time calibration, stone observatories, sun-dials.

TIME-KEEPING has been very significant in the scientific as well as socio-cultural traditions around the world ${ }^{1,2}$. Alfred Gatty ${ }^{3}$ has nicely described this fact at length with documentation of numerous sun-dials of different timeperiods in history.

Sun-dials and time-keeping instruments have played an important role in the development and evolution of astronomy ${ }^{3-6}$. Various workers have documented the heritage of stone observatories and a variety of time-keeping instruments in a comprehensive manner ${ }^{4-8}$. Still there are many sun-dials with different styles and concepts created in different periods in the past that have yet not been documented $^{2}$.

In continuation of previous studies ${ }^{2}$, we report here two very different sun-dials situated in Srirangapatna. One dial is at Gumbaz (Figure 1) that is Hyder Ali's tomb and the other is situated inside Jamia Masjid (Figure 2) ${ }^{9}$.

These sun-dials have braved the sun, rains and the winds over a couple of centuries and have survived all odds with little weathering effects visible on them. These days, the sun-dials at both the monuments are being maintained by the Archaeological Survey of India (ASI). It was Tipu Sultan who got the sun-dials installed on these two locations. Both the sun-dials are engraved on granite slabs. The sun-dial on the Jamia Masjid's terrace has engravings on a near circular slab, whereas the one near Gumbaz is engraved on a square slab. There are eight equally spaced radial lines that intersect at the centre on the dials. The sun-dial at Jamia Masjid is

\footnotetext{
*For correspondence. (e-mail: aalok.pandya@jecrcu.edu.in)
}

mounted on a small platform next to the southern entrance on the first floor. In both the sun-dials there are cavities at the centre to erect a small vertical pole as a gnomon (Figure 1).

The north-south line of the Gumbaz dial is misaligned by about eleven degrees towards the east. The dial's rectangular granite slab measures $63 \times 48 \mathrm{~cm}^{2}$ (Figure 2).

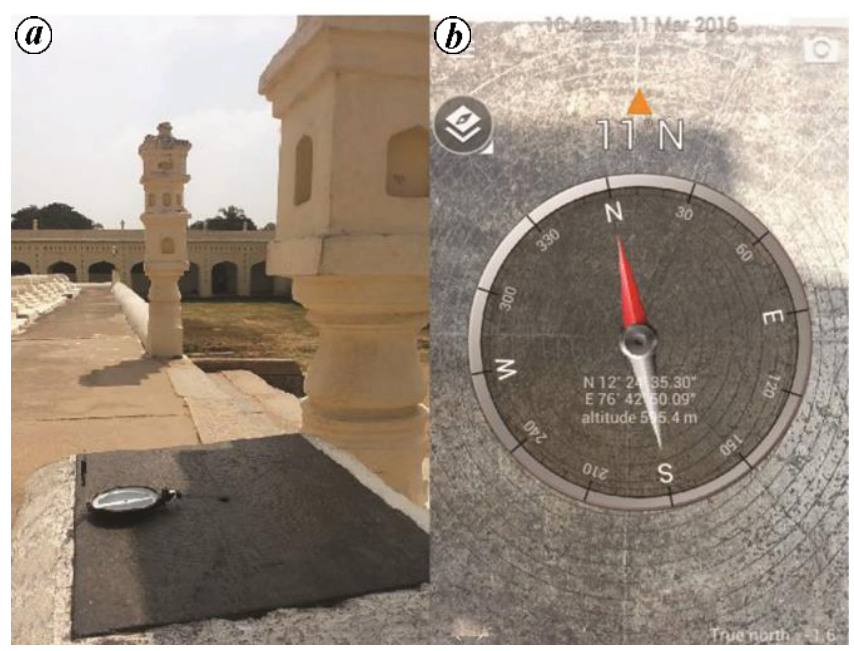

Figure 1. $\boldsymbol{a}$, The sun-dial at Gumbaz in Srirangapatna; $\boldsymbol{b}$, Top view of the sun-dial in Gumbaz using smart phone applications of compass and GPS.

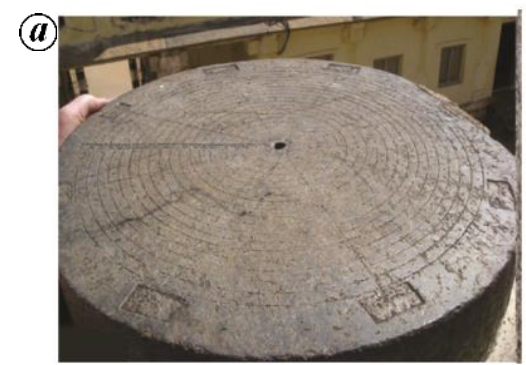

(b)

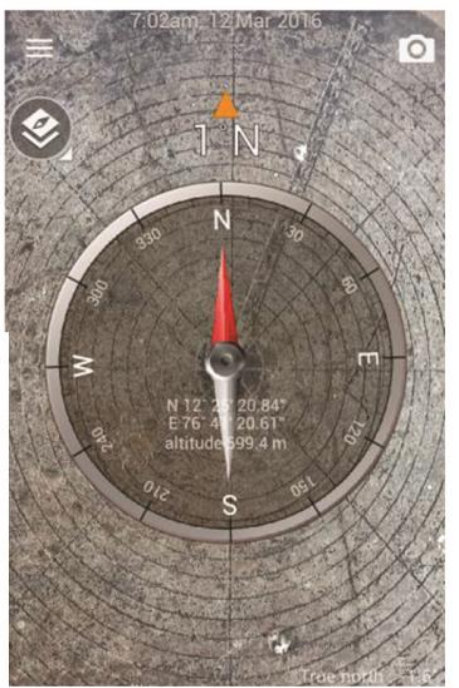

Figure 2. $\boldsymbol{a}$, The sun-dial in Jamia Masjid; $\boldsymbol{b}$, Top view of the sundial in Jamia Masjid using smart phone applications of compass and GPS. 


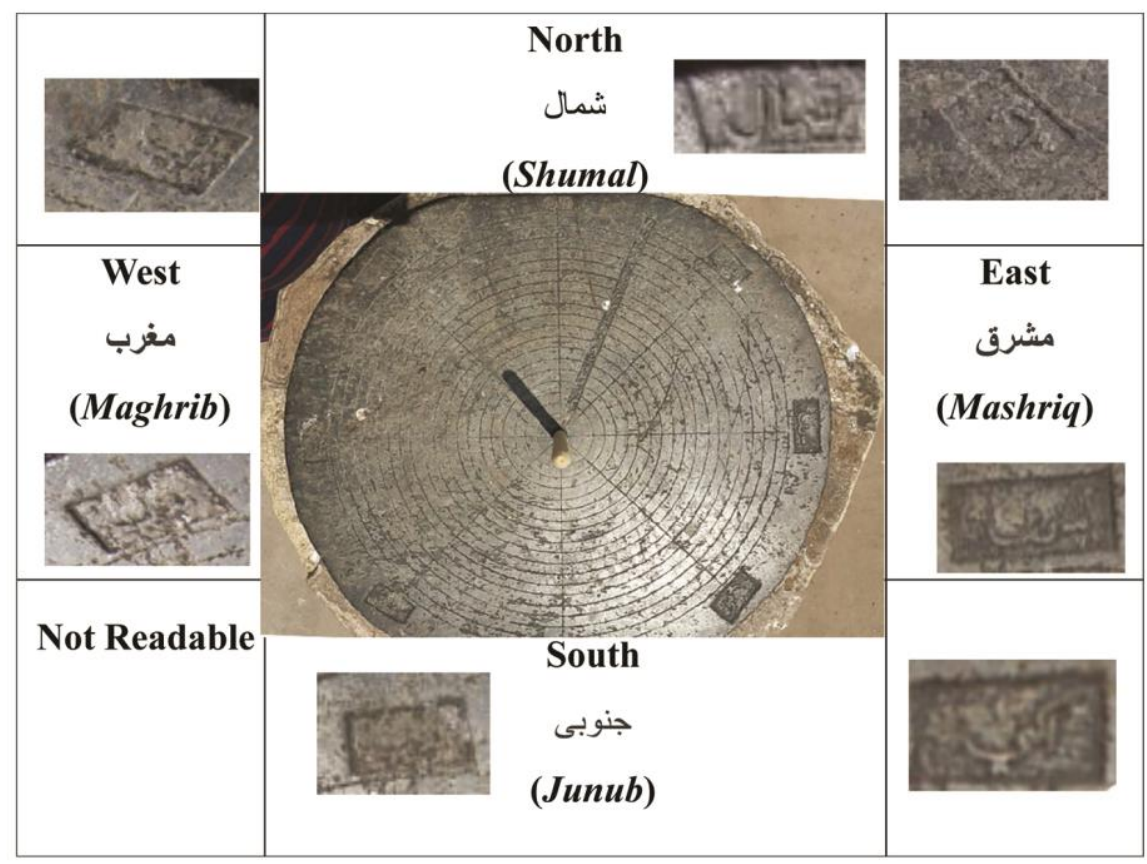

Figure 3. Inscriptions on the sun-dials.

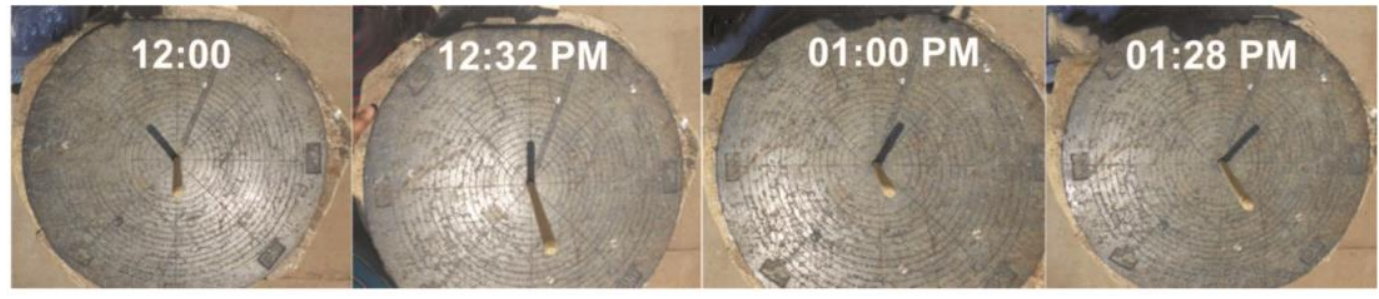

Figure 4. Photographs of the observations recorded on 11 March 2016.

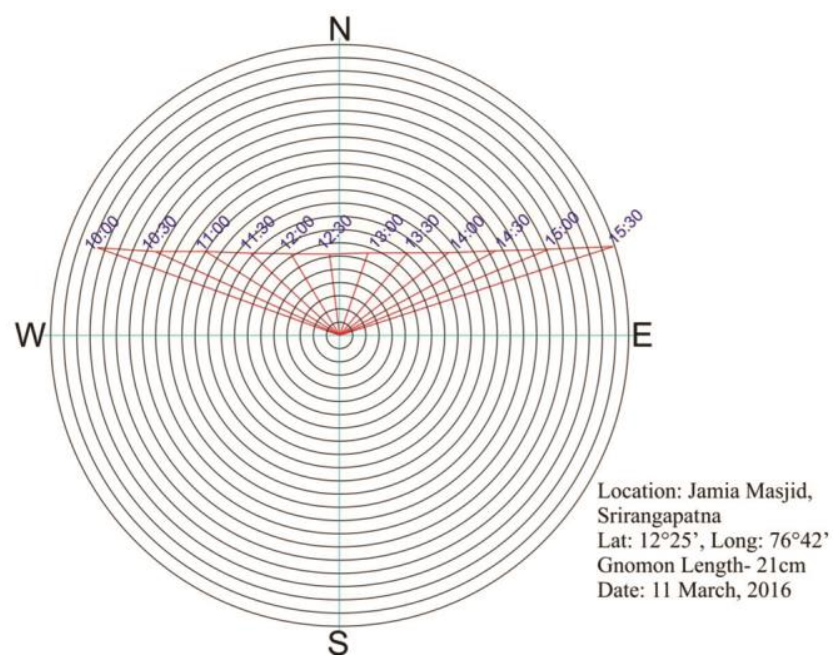

Figure 5. Computer simulation of variation in shadow on 11 March (gnomon length $21 \mathrm{~cm}$ ).

All the inscriptions on the dial are in Persian. The directions are prominently inscribed as Shumal (north), Junub (south), Mashrique (east) and Maghrib (west). Some of the inscriptions are not readable (Figure 3).
The dial at Jamia Masjid is better aligned compared to the one at Gumbaz. The deviation of the meridian line on this dial is just about a degree towards the east. A $1 \mathrm{~cm}$ wide radial strip close to the north-south line is engraved with the order of the twenty concentric circles on the dials. The numbers are barely readable. The construction of Jamia Masjid ${ }^{10}$ itself was completed around AD 1787. So the sun-dial, which is ensconced on the top of a small platform on the first floor of the mosque, belongs to that era. Soon after these installations, Tipu Sultan acquired a modern clock from France. Once the French clock came in regular use, sun-dials became redundant. The other salient features of these sun-dials could be summarized as follows: (i) There are 20 concentric circles in addition to the central cavity meant for erecting a gnomon. The diameter of the cavity at the centre is $1 \mathrm{~cm}$ and that of the first circle is $2 \mathrm{~cm}$. Radius of each consecutive circle differs from its adjacent one by $\sim 1 \mathrm{~cm}$. (ii) There are no angular graduations on the dial to measure the intervals of time as is usually found in other sun-dials. Thus, we ought to analyse this sun-dial in an altogether different perspective. However, we can verify the shortest shadow 


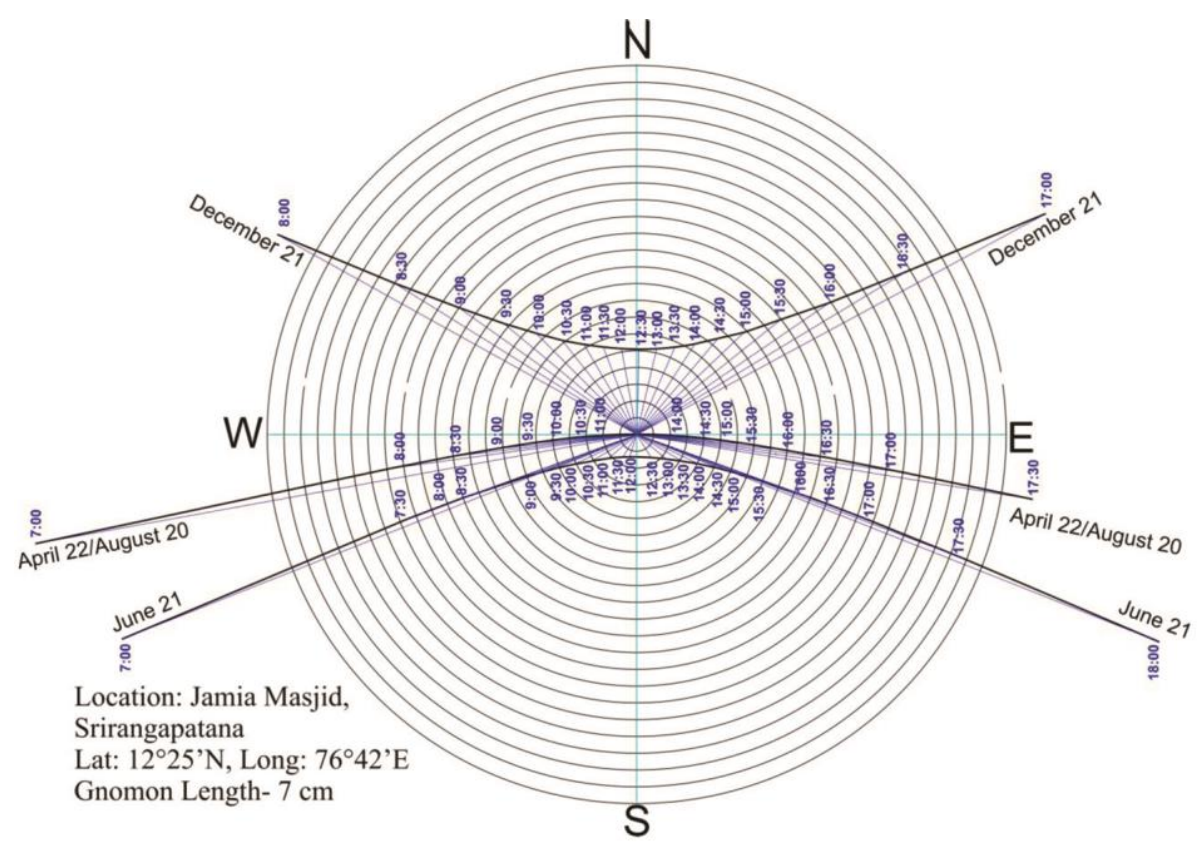

Figure 6. Computer simulation of the variation in shadow length on 22 April, 21 June, 20 August and 21 December (for gnomon length $7 \mathrm{~cm}$ ).

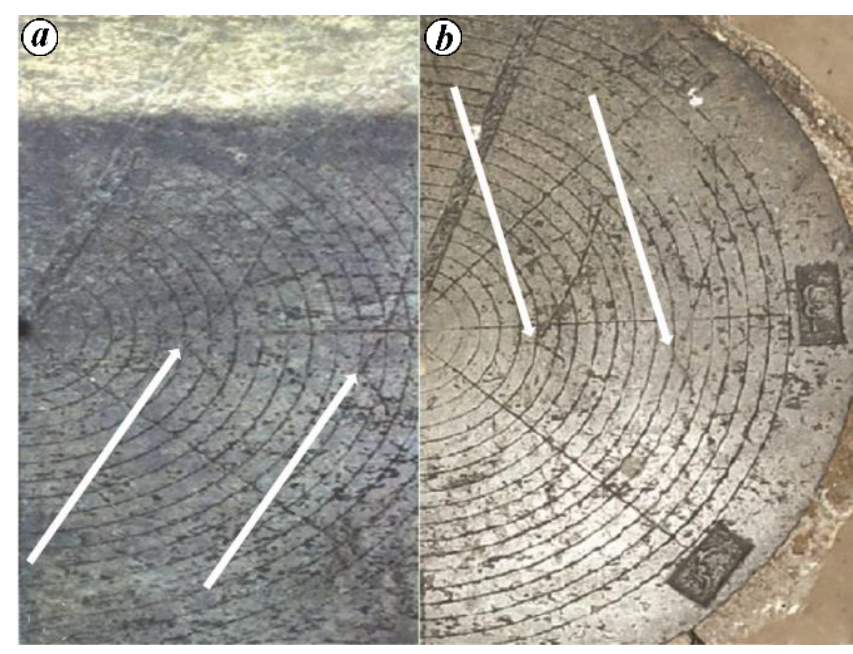

Figure 7. Two curves with their tips at the 7 th and 14 th circles respectively on the sun-dials: (a) Cusps on the sun-dial in Gumbaz; and (b) Cusps on the sun-dial in Jamia Masjid.

of the gnomon at meridian on the sun-dial as a standard test. We planted a prefabricated $21 \mathrm{~cm}$ long gnomon that fitted well in the central cavity of the dial and recorded our observations on 11 and 12 March 2016. (iii) There is a difference of $82.5^{\circ}-76.88^{\circ}\left\{5.52^{\circ}\right.$ between the longitudes of Srirangapatna and central meridian of India. Thus, the local time of Srirangapatna differs from IST by $5.52 \times 4=22.08 \mathrm{~min}$. There was an additional correction of $-9.95 \mathrm{~min}$ on $11 \mathrm{March}$ and $-9.68 \mathrm{~min}$ on $12 \mathrm{March}$ due to equation of time. Hence, the local noon was observed on 11 and 12 March at 12:32 IST. We verified the incidence of meridian at respective local time.
We computed the shadow lengths at Srirangapatna for various hours from the ephemeris data and plotted the same on a simulated dial for the same day. Evidently, the plotted data and the observations are in agreement. These observations that appear in successive intervals are shown in Figure 4. The plot of the computer generated data for the same date matches the observations (Figure 5).

We also computed the locus of the gnomon's shadow tip during three additional astronomically crucial dates for the place, viz. 21 December, 21 June (two solstices), and on 22 April and 20 August (dates of Sun's zenithal passage dates over Srirangapatna) (Figure 6).

We also observed two intriguing curves inscribed on the dials that are geometrically prominent cusps. The tips of the cusps touched respectively seventh and fourteenth circles of the sun-dial. Interestingly, these curves are inscribed on both the sun-dials of Srirangapatna (Figure 7).

These sun-dials came in handy for people who wanted to ascertain the times for prayers in the absence of a mechanical watch. There was a practice of keeping sun-dials in places of worship in the past. Based on the shadow that the gnomon casts on a calibrated dial, particular prayer time can be determined. The guidelines for prayers in Islam depend on the movement/position of the sun and geographical location.

However, the prescriptions of prayer times are not universal as the location of a place is also an important factor in the determination of prayer time and adjustments are to be made for higher latitudes and specific locations ${ }^{11}$.

The tips of the cusps on the dial give us a clue for this exercise. The incidence of tip of the cusp on the seventh 


\section{RESEARCH COMMUNICATIONS}

circle could be used to standardize the gnomon length for the given sun-dial. This hypothesis is validated at the end of the study. We thus expect the tip of shadow of gnomon to coincide with seventh circle for a $7 \mathrm{~cm}$ long gnomon on a particular day.

We developed a computer program in FORTRAN to calculate the shadow lengths for various times of a day throughout the year. We performed this exercise for different days of a year considering at least three days of a month and also for two equinox days, two solstices and 22 April and 20 August when the sun is overhead at Srirangapatna during its upper meridian transit. We note that on 22 April and 20 August close to 3:30 p.m., the shadow length of a $7 \mathrm{~cm}$ long gnomon reaches the seventh circle, that is when the length of the shadow becomes equal to the length of the gnomon itself. The results of our computations were impressive. In fact, if we track the locus of the shadow tip at a fixed time say 3:30 p.m. every day, this results into the shape of an 'analema' (Figure 8).

However, the engraved curves on the sun-dial appeared as dissected and discontinuous half analema. The curves follow only the convex parts of the analema. Why on earth only half analema was considered by the dial's designers?

We further try to understand the entire exercise in the light of practice of prayer guidelines across the world. In the calculation of prayer times, we make use of the expression of hour-angle (HA) of the sun and the time at which the sun reaches an angle $a$ above the horizon which is given by the formula

$$
\mathrm{HA}=\frac{1}{15} \arccos \left(\frac{\sin (a)-\sin (\varphi) \sin (\delta)}{\cos (\varphi) \cos (\delta)}\right),
$$

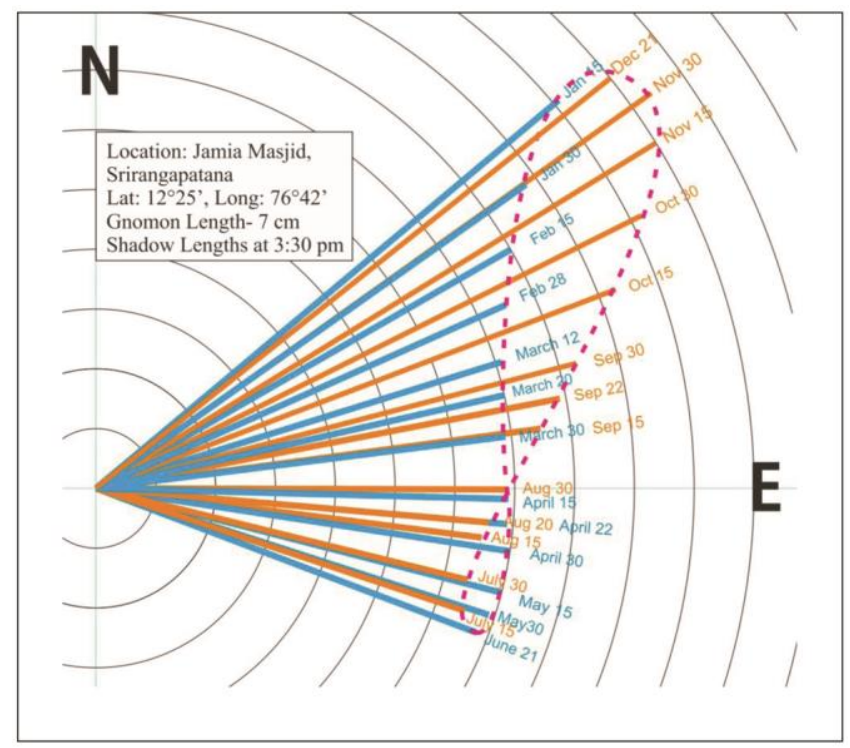

Figure 8. Variation of shadow length at a fixed time on different days: an analema. where $\varphi$ denotes latitude of a place, $\delta$ denotes sun's declination and $a$ denotes altitude of the sun above the horizon such that $a=0$ at sunrise and sunset.

There are more than one prescriptions to calculate the prayer time of the late afternoon prayer (ASR) which various schools of thought follow addressing seasonal variations and local geographical conditions.

We thus revisit the criteria for the prayer time of ASR. We find an additional prescription for the calibration of prayer times for a given location. According to this criterion, for the gnomon length of $7 \mathrm{~cm}$, we prescribe the time for beginning of the prayer of ASR as the time when the length of the shadow is equal to ' $7 \mathrm{~cm}+$ length of the shadow at local noon the same day'. The time for the end of the prayer of ASR is prescribed as the time when the length of the shadow is equal to ' $14 \mathrm{~cm}+$ length of the shadow at local noon on the same day'. In simple words, we ought to consider a correction factor that is 'length of the shadow at local noon on a given day' and add that in the calculation of two afternoon prayer times.

For the regions located between the equator and the Tropic of Cancer, the hour angle $\mathrm{HA}(k)$ for the prayer of ASR is prescribed as

$$
\begin{aligned}
\mathrm{HA}(k) & =\frac{1}{15} \operatorname{arc~cos} \\
& \left(\frac{\sin (\operatorname{arc} \cot (k+\tan (\varphi-\delta))-\sin (\varphi) \sin (\delta)}{\cos (\varphi) \cos (\delta)}\right) .
\end{aligned}
$$

So that $\mathrm{HA}(1)$ at $k=1$ denotes hour angle at the beginning of the prayer of ASR and HA(2) with $k=2$ denotes the hour angle for the end of the prayer of ASR. Where the expressions of altitude are given by

$$
a=\operatorname{arc} \sin \left[\sin (\varphi) \sin (\delta)+\cos (\varphi) \cos \left(15^{*} H A\right)\right],
$$

and azimuth corresponding to this particular hour angle could be given by standard expression

$$
A=\arccos \left(\frac{\sin (\delta)-\sin (\varphi) \sin (a)}{\cos (\varphi) \cos (a)}\right) .
$$

Using expressions in eqs (2), (3) and (4), the shadow length of the gnomon at the local noon could be calculated as

$$
X=7 * \tan (\varphi-\delta)
$$

Finally, the shadow length of the gnomon at the time of the prayer of ASR could be calculated as

$$
X(1)=7+7 * \tan (\varphi-\delta) .
$$




\section{RESEARCH COMMUNICATIONS}

Similarly, the shadow length of the gnomon at the end of prayer of ASR could be calculated as

$$
X(2)=14+7 * \tan (\varphi-\delta) \text {. }
$$

The shadow lengths $X(1)$ and $X(2)$ on the selected days of the year were calculated by a computer program. Using this data we draw computer graphics as shown in Figures 9 and 10.

Using the data from this computer program ${ }^{12}$, we plot effective shadow lengths indicating the beginning of the prayer time of ASR and the shadow lengths that indicate time for the end of the prayer.

The effective shadow lengths $X(1)$ and $X(2)$ are plotted at respective azimuth angles. In Figure 9, the tip of orange line indicates beginning of the ASR prayer and the tip of blue line indicates the end of ASR (Figure 10). Moreover, the locus of the tip of the shadow when plotted using our computed data mimics exactly the two cusps inscribed on the sun-dial.

Here, the first cusp indicates the beginning of third ASR prayer and the second cusp indicates the end of ASR.

Summarily, the sun-dial in a mosque could serve the purpose of an indicator for prayer as follows. The early afternoon prayer ZUHR should start just after the shadow reaches the meridian line and should end as the gnomon shadow reaches the first cusp (ASR-1). The third prayer should start soon after the gnomon shadow crosses the first cusp (ASR-1) and should end before it reaches the second cusp (ASR-2).

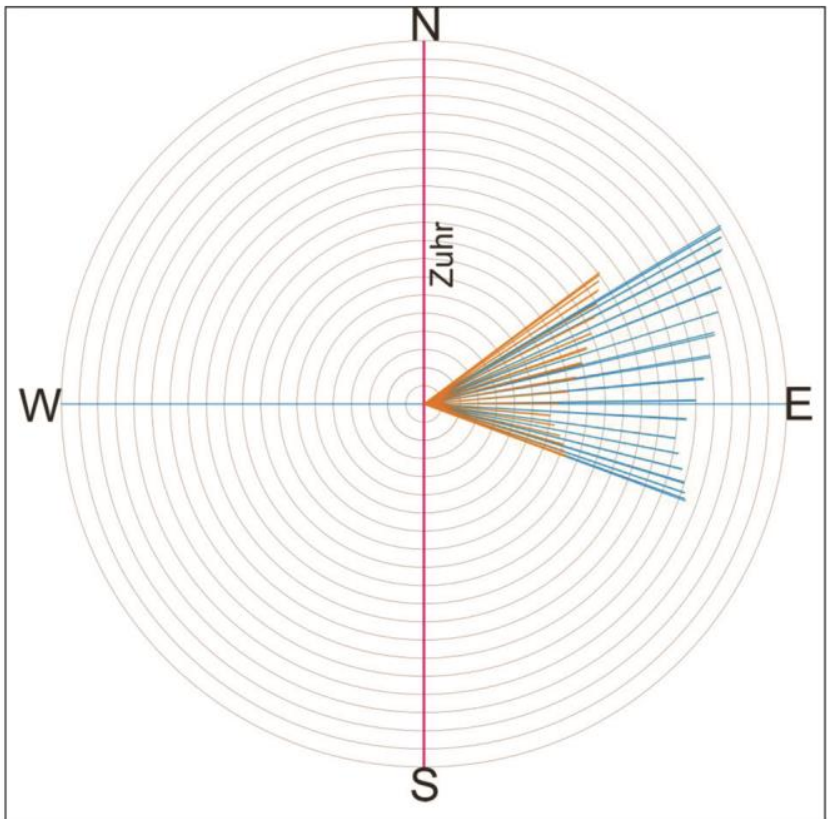

Figure 9. Shadow lengths on different days: from computed data.
We summarize the entire exercise by a simple graph based on the data generated (Figure 11).

The ending of ASR can also be computed similarly and the plot can be a scaled version of the present one. This plot amply explains why the single inflexion point of the cusps on the dial represents two separate dates (Supplementary Tables 1 and 2).

The sun-dials at Srirangapatna are indeed very different from all other sun-dials we have come across so far.

As there are tall trees in the east of Jamia Masjid, they cause long shadows in the morning. These days, the sundial becomes usable at around 10:30 a.m. and is useful till 2:00 p.m. After that, the sun hides behind the structure of the mosque in the west. It is learnt that the mosque did not have first floor during the period AD 1782-1796, and the dial could be used till late afternoon back then.

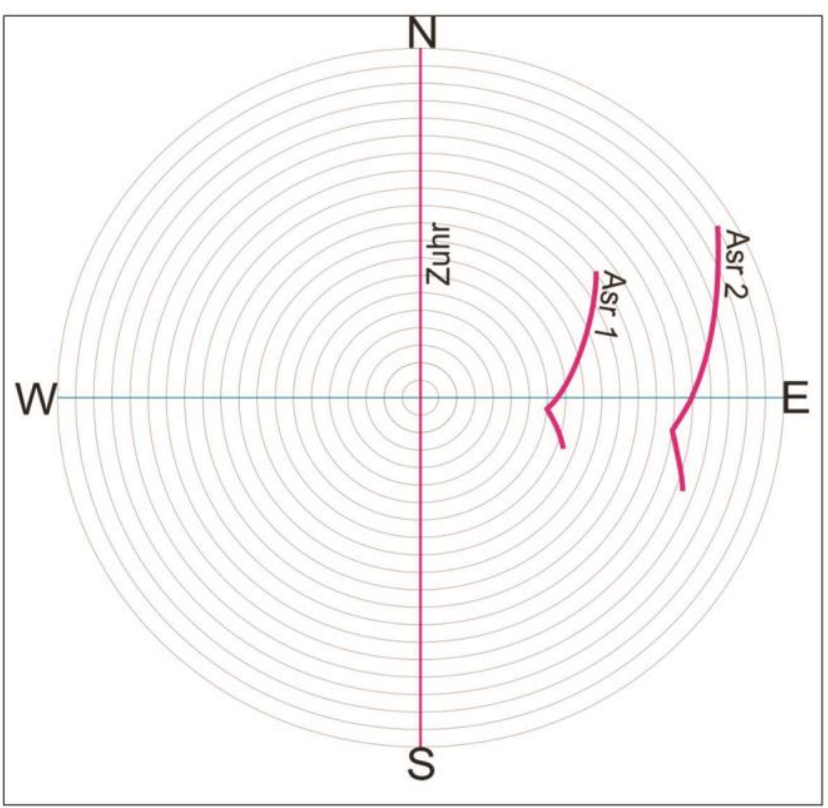

Figure 10. Computer simulation of the locus of the tip of the shadow on different days.

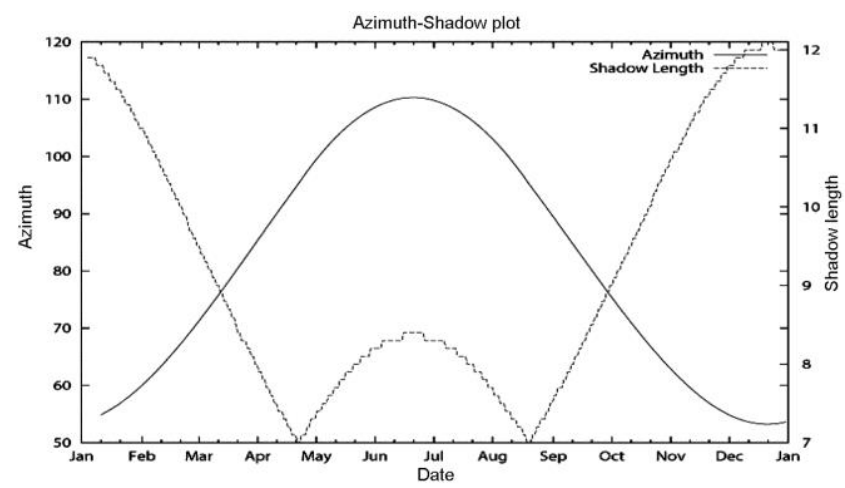

Figure 11. A graph plotted from the computed data for the beginning of ASR on different days of a year. 
We faced similar problem while observing the sun-dial at Hyder Ali's tomb. One of the minarets erected later in the neighbourhood blocks incidence of sun light on the dial for a significant duration of the day. This problem is obvious in Figure $1 a$ also.

1. Balasubramaniam, R. and Dass, M. I., On the astronomical significance of the Delhi Iron Pillar. Curr. Sci., 2004, 86(5), 1134-1142.

2. Pandya, A., Bahadur, T. and Bhattacharya, S., Time keeping in the past: sun-dial in Jaisalmer Fort. Indian J. Hist. Sci., 2017, 52(2), $138-147$.

3. Gatty, A., The Book of Sun-Dials, George Bell and Sons, London, 1872, pp. 1-2; http://digital.library.upenn.edu/women/gatty/ sundials/sundials.html

4. Kaye, G. R., The astronomical observatories of Jai Singh, Arch. Surv. India, New Imperial Series, 1973, XL.

5. Singh, P., Indian and Islamic Stone Observatories, Holiday Publications, Jaipur, 2009

6. Singh, P., Stone Observatories of India, Bharat Manisha, Varanasi, 1975 .

7. Kochar, R. and Narlikar, J., Astronomy in India, Indian Nationa Science Academy, New Delhi, 1994, pp. 6-9.

8. Kochar, R. and Narlikar, J., Astronomy in India: Past, present and future, Inter University Centre for Astronomy and Astrophysics, Pune, and Indian Institute of Astrophysics, Bangalore, 1993, pp. 11-18.

9. Heritage of the Mysore division, The District Gazetteers of Mysore and Mandya, Deptt. of Gazetteer, Bangalore, http://rcmysore. gov.in/heritage.pdf

10. Sheik Ali, B., Tipu Sultan: A Crusader for Change, Knowledge Publication Society, Mysuru, 2014, pp. 390-391; www.bshekali.in

11. Abdul Karim, S. M., A Guide to PRAYER in Islam, The Cooperative Office for Call and Foreigners' Guidance at Sultanah, Riyadh, 1997, pp. 16-17; http://www.islamicbook.ws/english/ english-020.pdf

12. For computer programme to calculate prayer time of Asr: http://astronomyaardra.org/res.htm

ACKNOWLEDGEMENTS. We thank the Archeological Survey of India (ASI), Bangalore Circle, and the authorities of Jamia Masjid and Gumbaz at Srirangapatna for permission. We also thank Prof. B. Sheik Ali and Prof. Karimuddin for explaining the rationale of a sun-dial in a mosque and the history of Jamia-Masjid and the Hyder Ali's tomb in Srirangapatna.

Received 4 April 2017; revised accepted 16 January 2019

doi: $10.18520 / \mathrm{cs} / \mathrm{v} 116 / \mathrm{i} 5 / 811-816$

\section{Impact of Cartosat-1 orography in $330 \mathrm{~m}$ Unified Model forecast}

\author{
A. Jayakumar*, Jisesh Sethunadh, \\ Timmy Francis, Saji Mohandas and \\ E. N. Rajagopal
}

National Centre for Medium Range Weather Forecasting, Ministry of Earth Sciences, Government of India, A-50, Sector 62, Noida 201 309, India

The newly introduced high-resolution $(330 \mathrm{~m})$ regional model, Delhi Model (DM), at the National Centre for Medium Range Weather Forecasting targets winter time fog/visibility forecast over Delhi, India. The present study focuses on the benefits of enhanced orographic features in DM, through a new data set developed using the Indian Space Research Organisation Cartosat-1 orography (Cartosat-run), against those from the NASA Shuttle Radar Topography Mission Digital Elevation Model employed previously (SRTMrun). The early morning visibilities from the Cartosatruns were lower compared to the SRTM-runs, which could be linked to an enhanced downdraft (negative vertical velocity) in the former, helping form a shallow and stratified boundary layer. The evolution and variability of 'ventilation index' in the model domain is regulated by the local wind circulation changes within the shallow boundary layer which in turn is modulated by the orography representation. The DM forecasted ventilation index has been projected to be a potential indicator of the atmosphere dispersion of airborne pollutants over Delhi.

Keywords: Cartosat, fog, orography, visibility.

SOCIO-ECONOMIC vulnerability to the poor visibility conditions in Delhi under extended and heavy fog spells during winter period exacerbates with the exorbitant conditions of poor air quality. Observational study by Ghude et al. ${ }^{1}$ shows that the increasing trend in fog days/hours over Indian cities is linked with enhanced urban aerosol loading and moisture availability. Conversely, the urban heat island (UHI) effect manifests itself as a gradient in the aforementioned fog trends between urban and suburb regions ${ }^{2}$. The Government of India has initiated intensive ground-based measurement campaigns such as WIFEX ${ }^{1}$, with a view to developing improved fog/visibility forecast capabilities in varying spatio-temporal scales. Better representation of land-atmosphere coupling and various physical processes is crucial to improving the fog/visibility forecast skills ${ }^{3}$.

Here we have focused on improving visibility forecasting in the National Centre for Medium Range Weather Forecasting's (NCMRWFs) high-resolution Unified Model

*For correspondence. (e-mail: jkumar@ncmrwf.gov.in) 